\title{
Admittance Control for Robotic Loading: Underground Field Trials with an LHD
}

\author{
Andrew A. Dobson, Joshua A. Marshall, and Johan Larsson
}

\begin{abstract}
In this paper we describe field trials of an admittance-based Autonomous Loading Controller (ALC) applied to a robotic Load-Haul-Dump (LHD) machine at an underground mine near Örebro, Sweden. The ALC was tuned and field tested by using a 14-tonne capacity Atlas Copco ST14 LHD mining machine in piles of fragmented rock, similar to those found in operational mines. Several relationships between the ALC parameters and our performance metrics were discovered through the described field tests. During these tests, the tuned ALC took $61 \%$ less time to load $39 \%$ more payload when compared to a manual operator. The results presented in this paper suggest that the ALC is more consistent than manual operators, and is also robust to uncertainties in the unstructured mine environment.
\end{abstract}

\section{INTRODUCTION}

In this paper we document the tuning and evaluation of an admittance-based Autonomous Loading Controller (ALC) by using the Atlas Copco ST14 Load-HaulDump (LHD) machine in the underground mine shown in Figure 1(a). A smaller 1-tonne robotic loader was initially used for ALC development prior to the work reported in this paper. Diesel-hydraulic LHDs are used in underground mines to move fragmented rock (in mining muck) from draw points to ore passes or trucks,

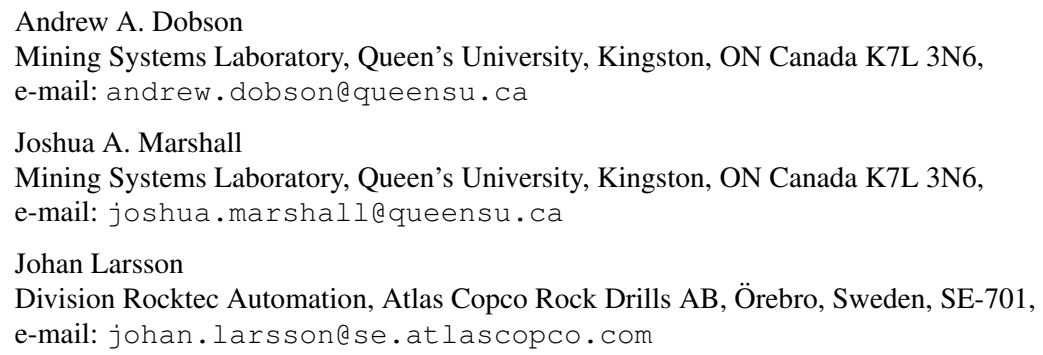


so the rock can be removed from the mine. Current robotic LHDs can haul and dump autonomously [1], but require an operator to load rock manually (usually by tele-remote). The ALC test results presented in Section 4 show a $39 \%$ increase in payload mass and a $61 \%$ reduction in dig time compared to an expert operator loading from the ST14 cab. The greater efficiency of the ALC over manual loading has implications for increasing mine productivity, and for decreasing costs by moving operators farther from potentially hazardous and remote mines [2].

Both the 1-tonne loader and the ST14 have similar hoisting and curling mechanisms as shown in Figure 1(b). Hoisting (vertical bucket motion) is controlled by altering the extension of the boom actuator, while curling (rocking the bucket forward and back) is controlled by altering the extension of the bucket actuator. The ALC admittance controller uses the forces sensed in the boom actuator to control the extension of the bucket actuator, and consequently, the curl of the bucket.

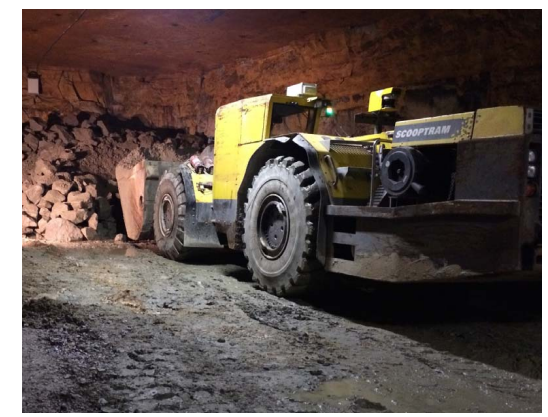

(a) Automated Atlas Copco ST14 LHD

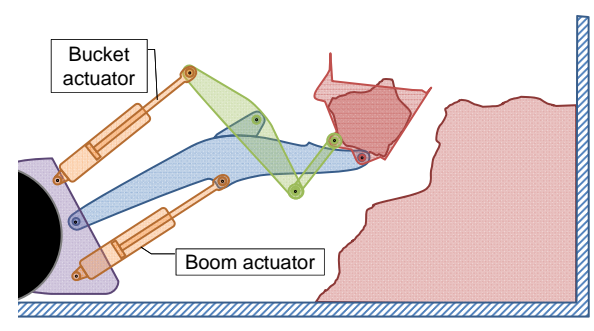

(b) Common hydraulic loading mechanism

Fig. 1 The ALC has been tested on a 1-tonne wheel loader (not shown), and a 14-tonne Atlas Copco ST14 LHD (a). Both vehicles use a boom and a bucket actuator to hoist and curl the bucket respectively (b). The ST14 field experiments described in this paper were carried out in an underground mine on a roadway consisting of a gravel and clay over a limestone subsurface.

Others have proposed using scripted dig paths, lookup tables, Artificial Intelligence (AI), and impedance control to automate the digging process. Many of these methods were tested in homogeneous materials (e.g., soil, sand, and gravel, but not fragmented rock) by using sub-scale excavators. The scripted and lookup table methods $[3,4,5,6]$ require pre-defined dig paths or bucket velocity targets, and did not perform well when sub-surface obstacles were encountered. The AI methods $[7,8,9,10,11]$ attempt to overcome this deficiency by using heuristically-derived digging rules, but these rules are generally difficult to develop and reproduce. These methods were also less efficient and consistent than human operators.

Impedance control $[12,13,14,15]$ is well-suited to tasks like trenching and landscaping, where the final target shape is more important than filling the bucket efficiently. This realization led Marshall in [16] to propose adapting Seraji's general admittance controller [17] for loading by controlling the admittance between the robot and the muck pile. Marshall's proposed admittance controller for loading was 
never tested, but was ultimately used as the starting point for the ALC presented in this paper. It is worth noting that despite a long history of research and development in robotic excavation, at the time of writing, there exists no widely-available commercial technology for autonomous digging in mining applications.

\section{ADMITTANCE-BASED AUTONOMOUS LOADING CONTROLLER (ALC)}

The proposed admittance controller modulates the error $e_{f}$ between a preselected target force $f_{T}$ and the sensed forces $f_{S}$ by altering the velocity $v_{A}$ of the bucket actuator, and consequently the bucket curl rate. In this way, the controller seeks to control the mechanical admittance $Y$ between the bucket and the muck pile, where

$$
Y=\frac{v_{A}}{f_{T}-f_{S}}=\frac{v_{A}}{e_{f}} .
$$

Intuitively, this approach is believed suitable for robotic loading in fragmented rock because a typical muck pile contains irregular rocks, having a range of sizes, with varying cohesion due to moisture content and other factors, which cause force variations as the bucket is moved through the pile. These conditions are not as suitable, for example, for path-tracking controllers where these disturbance cause large deviations from the desired path. Also, the muck pile itself is expected to comply during the excavation process unlike in impedance control where the robot complies to the target. For example, a window washing robot must comply to its target to prevent breaking the uncompliant glass. Hence the widow washing problem is better solved by using an impendence controller. When loading rock the opposite situation occurs since the target rock must comply to the motion of the bucket. This inverse compliance relationship makes the loading problem better suited to admittance control.

The admittance controller is implemented in one of the four states of the ALC finite state machine. Each state in the ALC is executed in order, as follows:

State 0 - Go to entry pose

State 1 - Drive into pile until entry forces are above entry force target

State 2 - Activate admittance controller until bucket has curled to breakout

State $3-$ Go to the weighing pose and terminate

Note that breakout occurs when the bucket curls past the point where additional material can easily enter the bucket, and is accompanied by a drop in digging forces [18]. In State 0, the ALC moves the boom and bucket to an appropriate entry pose before switching to State 1. In State 1, the LHD is commanded to drive forward until the bucket encounters enough resistance (as measured by the boom hydraulic cylinder pressures) to activate the admittance controller, at which point it switches to State 2. In State 2, the admittance controller controls admittance by referencing a target actuator force $f_{T}$. A high-level block diagram for this admittance control scheme is shown in Figure 2. While any controller $C$ could be used to map the 
force error $e_{f}$ to the actuator velocities, a simple proportional-type (P) admittance relationship was evaluated in the field experiments presented in this paper. In this instance, the constant admittance relationship is given by

$$
v_{A}=k_{A} \cdot e_{f},
$$

where $v_{A}$ is the actuator velocity, $k_{A}>0$ is the (admittance) gain, and the force error $e_{f}$ is given by $e_{f}=f_{T}-f_{S}$.

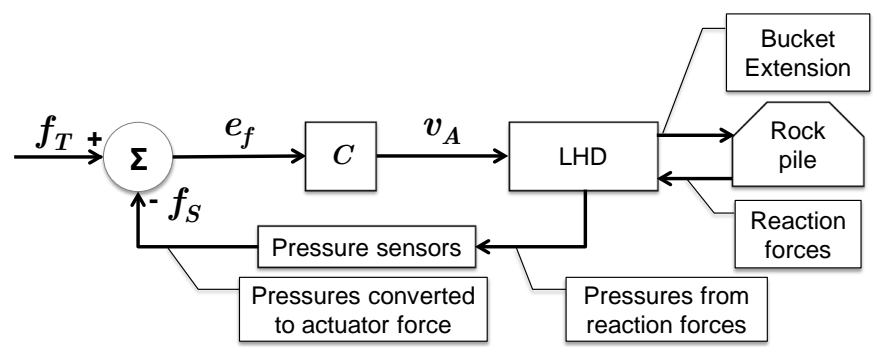

Fig. 2 The admittance controller uses any suitable controller $C$ to map the error between the desired and sensed actuator forces to the range of possible actuator velocities.

The bucket motion direction depends on both the reaction forces $f_{S}$, and the element used to sense $f_{S}$. The ALC admittance controller alters the bucket actuator velocity by using the boom actuator to measure $f_{S}$. In a conventional admittance controller the actuator velocity is controlled by using the forces sensed in the same actuator. We use the forces sensed in the boom actuator because 1) the actuator loading in Figure 3 shows that the boom actuator will tend to sense increasing forces as the bucket is curled up, 2) the boom stops on the ST14 tend to unload the boom actuator, which biases the ALC toward the breakout condition, and 3) the boom forces were generally cleaner than the bucket forces (see Figures 5, 6, and 8).

In Figure 3(a), curling up tends to decrease the forces sensed in the bucket actuator, while increasing the forces sensed in the boom actuator. When $f_{S}$ decreases $e_{f}$ increases, which causes the admittance controller in Equation (2) to respond by increasing $v_{A}$ until the bucket actuator velocity limit is reached. Reaching the velocity limit saturates the ALC, which means the ALC can no longer control the admittance between the bucket and muck pile. Hence it is better to sense $f_{S}$ in the boom actuator since curling up increases boom loading, which decreases $e_{f}$ and hence $v_{A}$. However, reducing $e_{f}$ to zero is also not desirable since this condition will result in no bucket velocity and no breakout. This situation is prevented in part by selecting an $f_{T}$ above the highest $f_{S}$, which ensures that $e_{f}>0$ as discussed in Section 4.1. Stalling is also prevented because the boom arms tend to be driven downwards as the bucket fills. This downward motion is eventually arrested by two boom stops. Once these stops are encountered part of the load flowing through the boom actuator is redirected through the boom stops, which tends to decrease $f_{S}$, and increase $e_{f}$ and $v_{A}$ right at the end of the dig when it is most beneficial for ensuring breakout. 


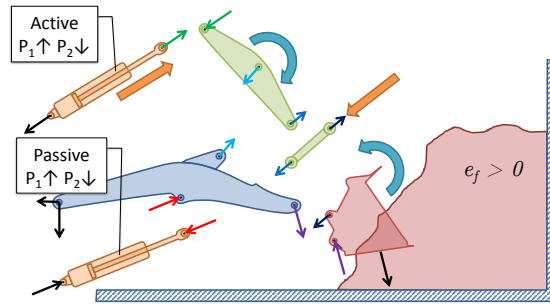

(a) Curl up

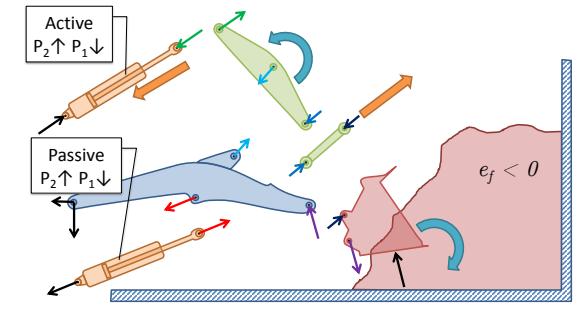

(b) Curl down

Fig. 3 When the bucket actuator extends in (a) $P_{1}$ goes up and the bucket curls back. The pile resists by putting the boom actuator in compression, which increases $P_{1}$ and the sensed reaction force. When the bucket curls down in (b) the inverse load case occurs and the boom actuator experiences tension. This tension manifests as a force drop because $P_{2}$ increases relative to $P_{1}$.

The digging forces are generated by both the bucket motion and the forward thrust of the LHD. When the forces sensed in the boom actuator are below $f_{T}$ the admittance controller will increase $f_{S}$ by curling up. Curling up increases the sensed forces in the boom because the boom actuator experiences compression in addition to the compression caused by the load in the bucket. Curling down tends to relieve this compression, which reduces $f_{S}$.

State 2 terminates when the bucket has curled passed the point where rock can easily enter the bucket (i.e., the breakout condition). Once breakout has occurred, the controller switches to State 3 where the LHD stops thrusting into the pile, raises the boom to the weighing pose, and finishes curling the bucket to settle the load. Once the dig cycle is complete, the dig time, total actuator work, and final payload are computed to determine dig efficiency.

\subsection{Dig Efficiency}

We define overall dig efficiency $\varepsilon_{d}$ as

$$
\varepsilon_{d}\left(t_{d}, W_{d}, M_{d}\right),
$$

which is a combination of three parameters: 1 ) the dig time $t_{d}$;2) the actuator work expended while digging $W_{d}$; and 3 ) the mass of rock in the bucket at the end of the dig attempt $M_{d}$. Together these three parameters define a point in 3D-space with time, work and mass axes (e.g., as shown in two 2D-space plots in Figure 7).

The payload mass $M_{d}$ was calculated by using a proprietary load weighing system described by Grahn [19]. This load weighing system calculates $M_{d}$ by

$$
M_{d}=k \cdot\left(P_{C}-P_{R}\right),
$$


where $P_{C}$ and $P_{R}$ are the boom actuator cylinder and rod pressures, and $k$ is a calibration constant for a specific weighing pose. According to Grahn, the load weighing system is calibrated to a precision of $\pm 0.5 \mathrm{t}$. The average ALC payload was $14.47 \pm 1.09 \mathrm{t}$, and the rated payload limit for the ST14 is $14 \mathrm{t}$.

Work and dig time are calculated between entry (after the entry force target is reached), and breakout. Let $n$ be the total number of sensor readings and let the subscript $i$ denote the time index associated with each sensor reading. Thus, the total work $W_{d}$ was estimated by

$$
W_{d}=\frac{1}{2} \sum_{i=1}^{n-1}\left[\left(F_{h, i}+F_{h, i+1}\right) \cdot\left|d_{h, i}-d_{h, i+1}\right|+\left(F_{c, i}+F_{c, i+1}\right) \cdot\left|d_{c, i}-d_{c, i+1}\right|\right],
$$

where $F_{h}$ and $F_{c}$ are the hoist and curl forces in the boom and bucket actuators respectively, and $d_{h}$ and $d_{c}$ are the displacements for each actuator. Note that this work estimate includes only the work done by the actuators, and not the drive train, which thrusts the loader into the pile.

\section{APPARATUS AND METHODOLOGY}

This section introduces the operating environment and test equipment used at the Kvarntorp Mine near Örebro, Sweden. Kvarntorp is an underground limestone room-and-pillar mine that is no longer in production. The test area is located approximately $30 \mathrm{~m}$ below surface, where the tunnels (called mine drifts) are approximately $10-12 \mathrm{~m}$ wide and $6 \mathrm{~m}$ tall. Over $200 \mathrm{t}$ of fragmented granite was added to the end of Drift 165 while the pile in Drift 159 consisted of several hundred tonnes of limestone from previous blasts in the mine. Drift 159 was primarily used for controller development and preliminary tuning, while Drift 165 was used for all manual digs and all final ALC digs. Figures 4(a) and 4(b) show the muck piles along the wall of Drift 159 and at the end of Drift 165 respectively. The largest visible dimension of the muck in Drift $159( \pm 1 \sigma)$ was $0.20 \pm 0.09 \mathrm{~m}$. The muck in Drift 165 was over twice as large, with double the standard deviation $(0.48 \pm 0.19 \mathrm{~m})$.

The Atlas Copco Scooptram ST14 is a $38 \mathrm{t}$ vehicle with a $14 \mathrm{t}, 6.4 \mathrm{~m}^{3}$ bucket. The nominal dimensions of the vehicle are $10.8 \mathrm{~m}$ long, $2.6 \mathrm{~m}$ tall, and $2.8 \mathrm{~m}$ wide [20]. The ST14 used for these tests was equipped for teleoperation [21]. However, the ALC only uses the actuator extension and pressure measurement sensors that are available on the stock ST14. The pressure measurements are taken on the rod and cylinder sides of the boom actuator. These pressures combined with the rod and cylinder areas ( $A_{R}$ and $A_{C}$ respectively) can be used to calculate $f_{S}$ by

$$
f_{S}=P_{C} \cdot A_{C}-P_{R} \cdot A_{R}
$$

Both the manual and final ALC dig trials were conducted at the end of Drift 165 in the granite muck pile. The actuator pressure and extension measurements were 


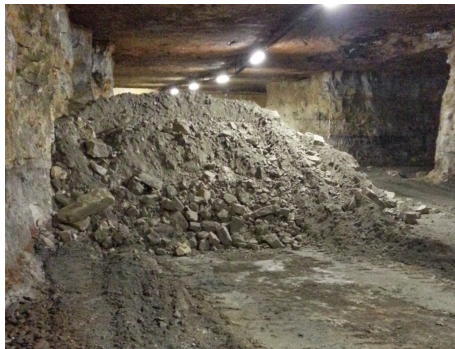

(a) Drift 159

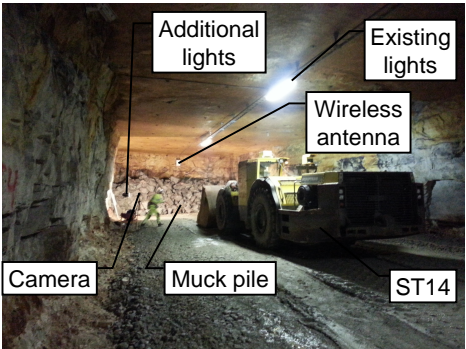

(c) Manual and ALC tests in Drift 165

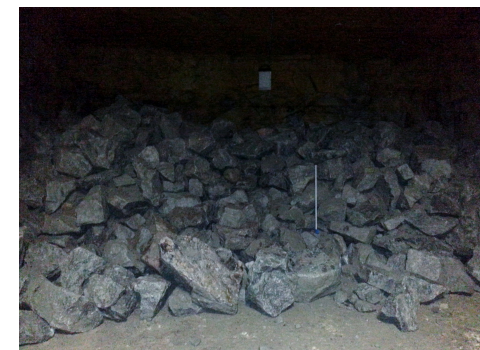

(b) Drift 165

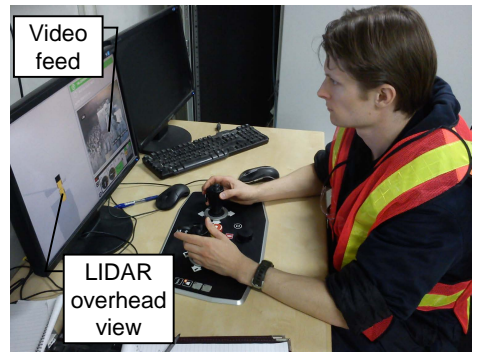

(d) Automation office control station

Fig. 4 The limestone muck pile along the wall of Drift 159 (a) was used for all preliminary logic tests and tuning, while the manual tests, final tuning, and ALC evaluation tests were conducted in the granite muck pile at the end of Drift 165 (b). The mean \pm one standard deviation rock size distribution estimates were $0.20 \pm 0.09 \mathrm{~m}$ in Drift 159 and $0.48 \pm 0.19 \mathrm{~m}$ in Drift 165 . (c) The ST14 began each dig in the start position, which was approximately $11 \mathrm{~m}$ from the toe of the pile. (d) The ST14 was moved into position by using the operator station within the automation office. Following automated loading, the operator weighed and dumped the material manually.

logged for both manual and autonomous operating modes, and were used to generate the digging histograms in Section 4. The vehicle was warmed up for 10 to $20 \mathrm{~min}$ at the beginning of each test day. Each dig began by positioning the vehicle in front of the muck pile as shown in Figure 4(c). For the manual dig trials, our expert operator "Frank" was instructed to dig 1) normally by using both boom and bucket actuators; 2) in a manor similar to the ALC using only the bucket actuator; and 3) by using $50 \%$ throttle. The $50 \%$ throttle setting was selected to determine if there were any advantages to digging at lower throttle. The manual dig efficiency results shown in Figure 7 indicate that digging at lower throttle should be avoided and hence the ALC throttle setting was set to $100 \%$ to better match the bucket only and both actuator manual digging methods. Similarly, the entry velocity was also selected to match the manual dig attempts and averaged $5.0 \mathrm{~km} / \mathrm{hr}$. This velocity corresponds to $100 \%$ throttle, first gear, and $0 \%$ brake.

In all tests, Frank controlled the vehicle from inside the ST14. The ALC digs began by switching the ST14 to "automation mode". The operator then left the vehicle, and entered the automation office shown in Figure 4(d). After uploading the desired 
tuning parameters to the ST14, the ALC was initiated. When the ALC reached its final state, the ST14 was switched to teleremote mode so that the bucket could be lifted, weighed, and dumped. The same weighing and dumping procedure was also performed by Frank following his dig attempts. After dumping, the ST14 was driven back to the approximate start position.

\section{FIELD EXPERIMENTS RESULTS}

The Autonomous Loading Controller (ALC) tuning tests were used to find final values for the ALC parameters, which were then held constant for all performance tests. These performance tests were conducted to compare the ALC to manual digging. The ALC parameters that were tuned were $f_{T}, k_{A}$, the breakout condition, as well as the entry and weighing poses. Additionally, field tuning revealed key information about controller saturation, ground detection, and ALC performance.

\subsection{Force Target $f_{T}$}

Figure 5 shows the ALC digging response as $f_{T}$ was reduced from $11 \mathrm{MN}$ to $9 \mathrm{MN}$. An initial guess for $k_{A}$ was 0.001 , which was selected by using

$$
k_{A} \approx r \cdot \frac{v_{A \max }}{f_{S_{\max }}}
$$

where $r=\frac{1}{8}, v_{A \max }$ is the maximum bucket actuator velocity $(0.08 \mathrm{~m} / \mathrm{s})$, and $f_{S_{\max }}$ is the maximum force sensed in the boom actuator (10 MN). $r$ is an arbitrary scalar that sets the minimum increment between no gain and a gain that results in complete actuator saturation. Initial tuning results (in Section 4.2) indicated that the controller was unacceptably saturated when $k_{A}$ was increased to 0.002 . Saturation should be avoided because it means the admittance controller is no longer maintaining the desired admittance dictated by Equation (2). The manual results (in Section 4.4 and specifically Figure 8) show that digging without compensating for the digging forces tends to result in less overall payload and more payload variability.

Decreasing the dig target increased dig time, decreased bucket velocity, and decreased bucket actuator control valve saturation. When $f_{T}$ dropped to $9.5 \mathrm{MN}$, the dig time increased from 8 to $30 \mathrm{~s}$, the bucket velocity was much slower, and the sensed forces were barely high enough to bias the admittance controller toward the breakout condition. At $f_{T}=9.0 \mathrm{MN}$ these effects became so severe that the dig failed because the ALC could then reduce the force error $e_{f}$ close to zero. Figures 5(a) and 5(b) also illustrate that more controller saturation leads to higher, more irregular forces. The 11-MN and $10-\mathrm{MN}$ test results indicate that these irregular forces generated higher payloads, but also more payload variability. It should also 
be noted that for the 11-MN and 10-MN tests the bucket curls down (see between 10 and $15 \mathrm{~s}$ ) when the boom forces exceed their respective $f_{T}$ values. While curling down may seem counter productive, it allows the bucket to circumvent force concentrations and dig deeper into the pile. We believe that this results in increased payload and less payload variability because the admittance between the bucket and muck pile is maintained, and hence each dig trajectory is tailored to the unique force environment encountered within the pile.

\subsection{Admittance Gain $k_{A}$}

Figure 6 shows the ALC responses when the admittance gain $k_{A}$ was raised from 0.001 to 0.002 while $f_{T}$ was maintained at $10 \mathrm{MN} . k_{A}=0.002$ was too high since the ALC valve commands were almost always saturated. $k_{A}=0.001$ was used for both the 10-MN and 11-MN performance tests, and was high enough to cover both positive and negative valve command ranges without saturation.

\subsection{Weighing Pose Entry Pose and Breakout Condition}

The weighing pose was set by eye such that the bucket was in free space above the pile. The entry pose was also set by eye such that the bucket was tilted downwards at approximately $15^{\circ}$ and scraping the floor. The breakout condition was set to $0.500 \mathrm{~m}$ of bucket actuator extension because the bucket is prevented from curling further by stops on the boom arms. However, as the boom rises these stops move further back. Midway through the tuning process, the bucket breakout extension was increased from 0.500 to $0.520 \mathrm{~m}$, which increased payload to $12.50 \mathrm{t}$ from $10.13 \mathrm{t}$. This increase occurred because the bucket curled back farther as soon as the boom started to lift, which kicked more material into the bucket. Only a few tests were performed at each breakout setting in the muck pile in Drift 159, before moving to the ALC performance tests. These performance tests were conducted in Drift 165, which contained the larger, higher density rock fragments. Several runs were made at both 10-MN and 11-MN dig targets and all other ALC parameters were kept constant so that the ALC could be compared to a manual operator.

\subsection{ALC Performance}

The dig efficiency results from the 26 autonomous and 28 manual digs are shown in Figure 7. The number of tests was dictated by the availability of the apparatus, operator, and test site. The manual digs with the highest dig efficiencies were Frank's bucket-only, and low-throttle digs. The autonomous digs with the highest efficien- 

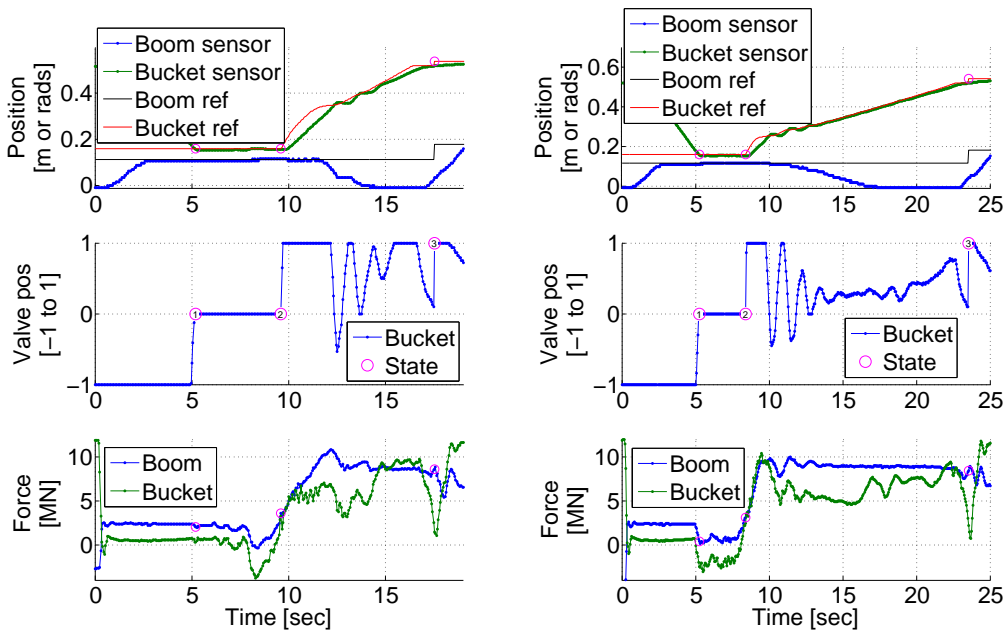

(a) $11.0 \mathrm{MN}$ dig target

(b) $10.0 \mathrm{MN}$ dig target
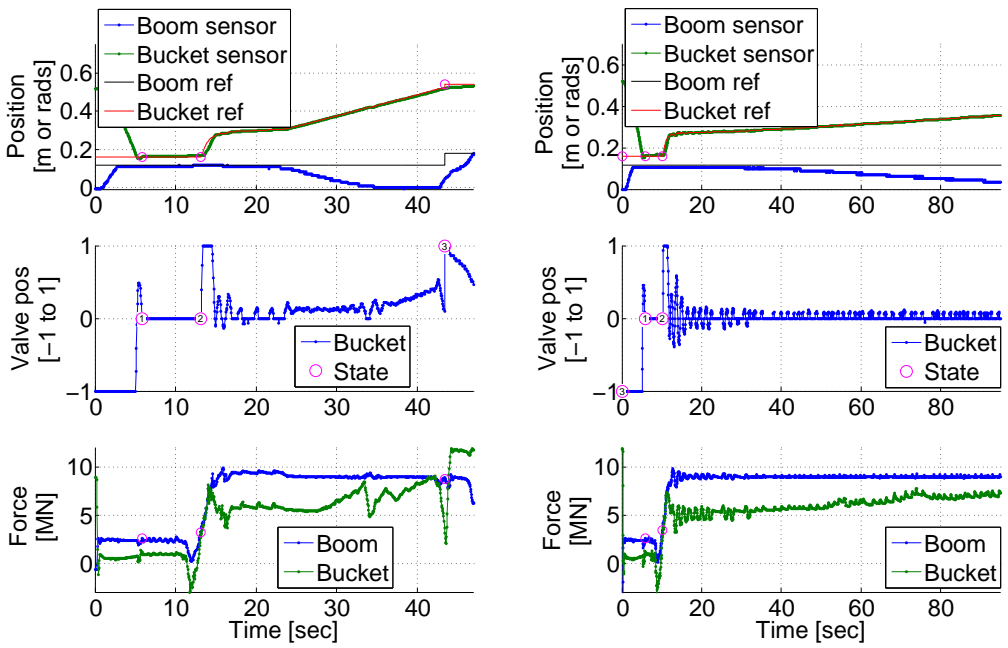

(c) $9.5 \mathrm{MN}$

(d) $9.0 \mathrm{MN}$ dig target

Fig. 5 Finding a dig force target - At $11 \mathrm{MN}$ (a) the ALC was more saturated than in the $10 \mathrm{MN}$ digs (b), but both completed successfully. The $10 \mathrm{MN}$ digs took twice as long as the $11 \mathrm{MN}$ digs, and the $9.5 \mathrm{MN}$ digs (c) took three times longer than the $11 \mathrm{MN}$ digs. At $9 \mathrm{MN}$ (d), the dig failed because the ALC was able to reduce the error to 0.0 and the curl rate dropped too low for the ALC to finish in a reasonable time. 

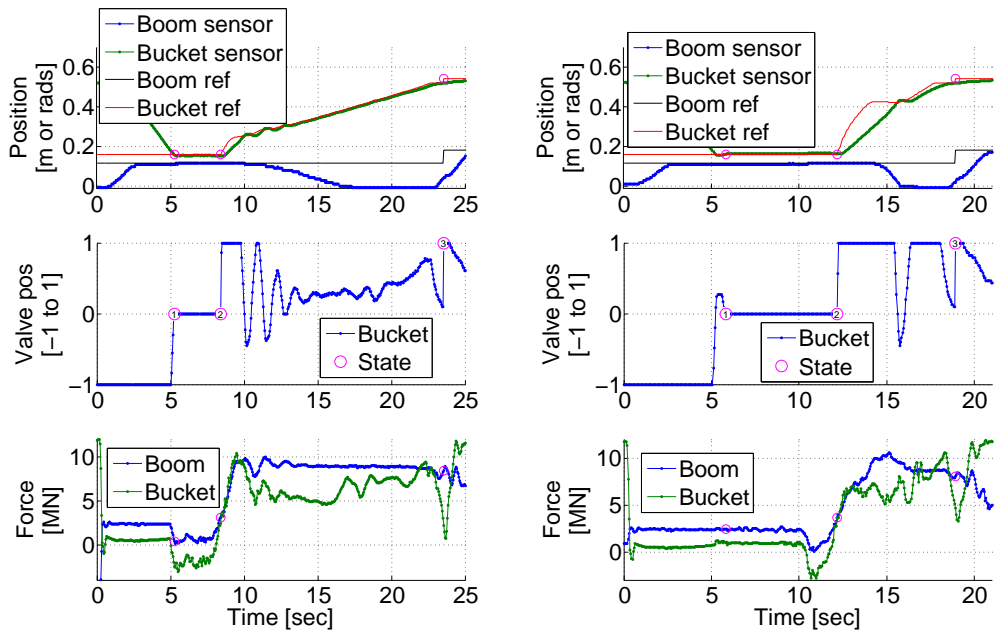

(a) ALC gain at 0.001 , Force target $10 \mathrm{MN}$

(b) ALC gain at 0.002 , Force target $10 \mathrm{MN}$

Fig. 6 ALC gain selection - The ALC gain at 0.001 (a) issues excellent valve commands with little saturation compared to the 0.002 gain (b), which was almost always saturated.

cies were the 11-MN digs. While the 10-MN autonomous digs were also excellent, six of these digs failed. The likely cause of these failures was low entry force due to striking the ground or spillage before entry.

Figure 7(a) shows the payload and dig times for the 54 dig attempts. While the autonomous dig attempts were tightly clustered, there was much more variability in the manual dig times and payloads. Figure 7(b) shows the payload and work expended for the same $54 \mathrm{dig}$ attempts. The autonomous dig attempts were again tightly clustered, while there was much more variability in the manual digs. Work also increased as payload increased. All dig efficiency results are summarized in Table 1.

Table 1 The ALC loaded $39 \%$ more payload in $61 \%$ less time, but required $68 \%$ more work than the best expert operator digs. The ALC was also much more consistent.

\begin{tabular}{lccc}
\hline$\varepsilon_{d}$ & \multicolumn{1}{c}{ Manual } & \multicolumn{2}{c}{ Autonomous Difference } \\
\hline$t_{d}[\mathrm{~s}]$ & $20.03 \pm 4.10$ & $7.82 \pm 0.26$ & $-61 \%$ \\
$W_{d}[\mathrm{MN} \mathrm{m}]$ & $2.59 \pm 1.17$ & $4.36 \pm 0.43$ & $+68 \%$ \\
$M_{d}[\mathrm{t}]$ & $10.41 \pm 1.77$ & $14.47 \pm 1.09$ & $+39 \%$ \\
\hline
\end{tabular}

Figure 8 shows the results for an excellent manual, and typical autonomous dig attempt. In both digs only the bucket was actuated either by Frank or by the 


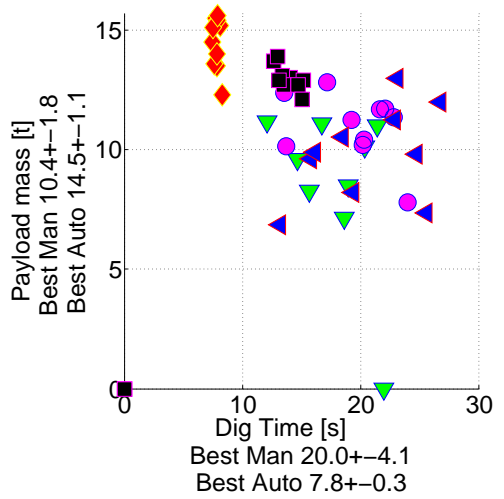

(a) Payload vs. Time

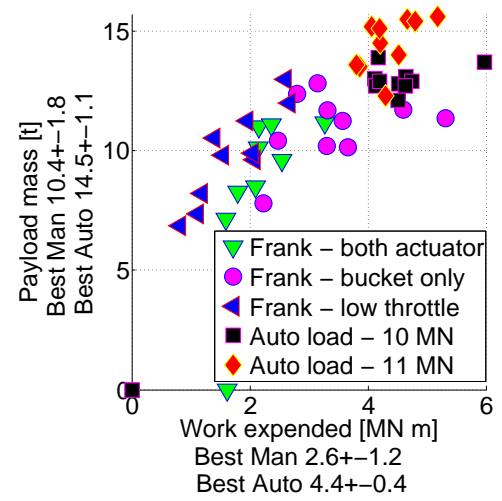

(b) Payload vs. Work

Fig. 7 The payload vs dig time (a) and the payload vs work (b) dig efficiency plots show that the only autonomous dig attempts that were less than $12 \mathrm{t}$ were the six $10 \mathrm{MN}$ dig target digs that failed due to insufficient entry force to trigger the admittance controller. The manual dig attempts had much greater variability in payload mass, dig time, and actuator work than the tightly clustered autonomous dig attempts. There is also a clear trend towards increasing work as payload increases (b).

ALC. Frank oscillated the bucket rhythmically while the ALC only oscillated when the forces were below the $11 \mathrm{MN}$ target force. This reduced oscillation resulted in smoother force and valve command profiles, and ultimately greater bucket velocity control, and more payload in less time.

Tests were conducted in both a settled and an unsettled muck pile, as well as the two muck piles with different rock types and size distributions. The average payload dropped from $14.47 \pm 1.09 \mathrm{t}$ in the unsettled pile (11-MN autonomous tests), to $12.50 \mathrm{t}$ in the settled pile. Only one test was performed in the settled pile since due to the time it takes for the pile to settle. The rock type and size distribution had little effect on the ALC because the force profiles resulting from digging in the two piles were nearly identical. The resulting payload change was slight, going from $11.40 \mathrm{t}$ in the lower density $0.20 \pm 0.09 \mathrm{~m}$ limestone rock in Drift 159 , to an average of $12.93 \pm 0.55 \mathrm{t}$ in the higher density $0.48 \pm 0.19 \mathrm{~m}$ granite rock in Drift 165 .

\section{CONCLUSION}

An Autonomous Loading Controller (ALC) based on constant admittance control was tuned and compared to manual dig trials at the Kvarntorp underground mine by using an Atlas Copco ST14 LHD, and various limestone and granite muck piles. In this paper, the admittance controller within the ALC prescribed a constant admittance relationship that used the forces sensed in the boom to alter the bucket 

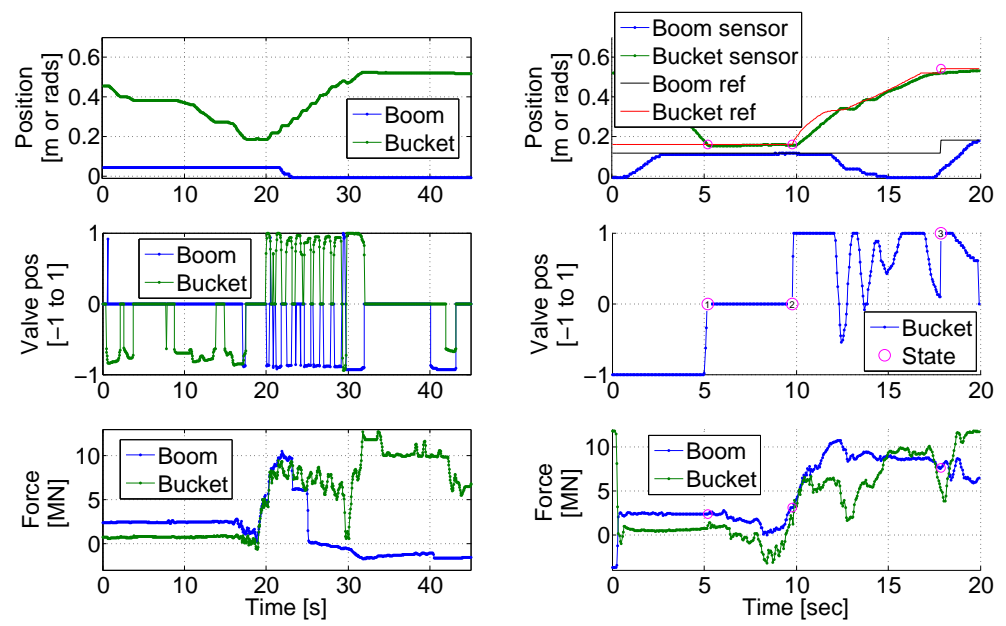

(a) Manual (Frank)

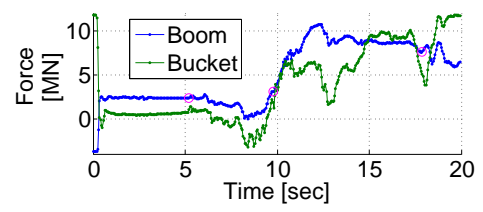

(b) Autonomous (11 MN)

Fig. 8 In (a) Frank gave the bucket regular oscillating command signals that resulted in a jagged force profile, and severe valve position oscillations between 1 and 0 . The ALC in (b) sent much smoother commands that used partial valve positions to regulate the speed of the bucket. As a result, the forces were much smoother than the manual dig attempts. Additionally the bucket curled down at $12 \mathrm{~s}$ to reduce the forces below the dig target. This behaviour caused the bucket to dig deeper into the pile, and ultimately increased the final payload.

velocity. Preliminary ALC tuning tests revealed that the dig target and admittance gain must be set such that the admittance controller can never fully reduce the force error to zero, which ensures that the ALC is biased toward breaking out of the muck pile. Biasing the ALC toward breakout made the ALC surprisingly robust to disturbances caused by changing much pile conditions. The performance comparisons between admittance-based and manual (expert operator) digs are the most important outcomes from these field experiments. However, vital insight was also gained into the digging process, as well as how to tune the ALC to match the machine to the test environment. The ALC had $61 \%$ better dig time and $39 \%$ greater payload, but required $68 \%$ more actuator work. The ALC dig efficiency variability was greatly reduced compared to the manual digs, which should make mass flow rates out of the mine easier to predict. Some 10-MN digs failed due to the uneven roadway. Ideally this variability in the roadway should be compensated for by maintaining a bucket hight relative to the ground.

Acknowledgements The authors would like to thank Atlas Copco Rock Drills AB for facilitating this research collaboration. This research was supported in part by the Natural Sciences and Engineering Research Council of Canada (NSERC) under grant 371452-2009. 


\section{References}

1. J.A. Marshall, T.D. Barfoot, J. Larsson.: Autonomous underground tramming for centerarticulated vehicles. Journal of Field Robotics 25(6-7), 400-421 (2008)

2. D. Zlotnikov.: Mining in the extreme. CIM 7(5), 50-56 (2012)

3. S. Sarata, N. Koyachi, K. Sugawara.: Field test of autonomous loading operation by wheel loader. in Proceedings of the IEEE/RSJ International Conference on Intelligent Robots and Systems, pp. 2661-2666. IEEE, Acropolis convention center, Nice, France (2008)

4. H. Almqvist.: Automatic bucket fill. Masters thesis, Linköping University, Linköping, Sweden (2009)

5. A.G. Shull. U.S. Patent No. 8160783.: Digging control system. (2012)

6. D.J. Rocke. U.S. Patent No. 5528843.: Control system for automatically controlling a work implement of an earthworking machine to capture material. (1996)

7. P. Lever.: An automated digging control for a wheel loader. Robotica 19(5), 497-511 (2001)

8. X. Shi, P. Lever, F. Wang.: Autonomous Rock Excavation: Intelligent Control Techniques and Experimentation. Series in intelligent control and intelligent automation, World Scientific, (1998)

9. A. Dasys, L. Geoffroy, A. Drouin.: U.S. Patent No. 5941921, Sensor feedback control for automated bucket loading. (1999)

10. D. Schmidt, M. Proetzsch, K. Berns.: Simulation and Control of an Autonomous Bucket Excavator for Landscaping Tasks. in IEEE International Conference on Robotics and Automation, pp. 5108-5113 (2010)

11. D.A. Bradley, D.W. Seward.: The Development, Control and Operation of an Autonomous Robotic Excavator. Journal of Intelligent and Robotic Systems 21(1), 73-97 (1998)

12. L. Bernold.: Motion and path control for robotic excavation. Journal of Aerospace Engineering 6(1), 1-18 (1993)

13. Q. Ha, M. Santos, Q. Nguyen, D. Rye, H. Durrant-Whyte.: Robotic excavation in construction automation. IEEE: Robotics and Automation Magazine 9(1), 20-28 (2002)

14. S. Salcudean, S. Tafazoli, K. Hashtrudi-Zaad, P. Lawrence.: Evaluation of impedance and teleoperation control of a hydraulic mini-excavator. Lecture Notes in Control and Information Sciences 232, 229-240 (1998)

15. G.J. Maeda, D.C. Rye, S.P.N. Singh.: Iterative Autonomous Excavation. in Field and Service Robotics Springer Tracts in Advanced Robotics, vol. 92, ed. by K. Yoshida, S. Tadokoro, pp. 369-382 Springer Berlin Heidelberg, Berlin Heidelberg Germany, (2013)

16. J.A. Marshall.: Toward autonomous excavation of fragmented rock: Experiments, modelling, identification and control. Master of science: Engineering, Queen's University, Kingston, On. Canada (2001)

17. H. Seraji.: Adaptive Admittance Control: an approach to explicit force control in compliant motion. in Proceedings of theIEEE International Conference on Robotics and Automation, vol. 4, pp. 2705-2712, (1994)

18. J.A. Marshall, P.F. Murphy, L.K. Daneshmend.: Toward autonomous excavation of fragmented rock: full-scale experiments. in IEEE Transactions on Automation Science and Engineering 5(3), 562-566 (2008)

19. F. Grahn.: Specification for load weighing. Internal T2 0330, Atlas Copco Rock Drills AB, Örebro Sweden (2005)

20. Atlas Copco.: Atlas Copco Underground loaders: Scooptram ST14 Technical specification. Atlas Copco (2012). Available ONLINE. http://www.atlascopco.ca/ images/technical_specification_scooptram_st14_9851_2350_01_ tcm836-1532858.pdf

21. J. Larsson, M. Broxvall, A. Saffiotti.: An evaluation of local autonomy applied to teleoperated vehicles in underground mines. in Proceeding of the IEEE Int. Conf. on Robotics and Automation, pp. 1745-1752 (2010) 